\title{
OPEN Biochar as a low-cost, eco-friendly, and electrically conductive material for terahertz applications
}

\author{
Woongkyu Park ${ }^{1,4}$, Hyuntae Kim ${ }^{2,4}$, Hajung Park ${ }^{2}$, Soobong Choi ${ }^{1,2 \bowtie}$, Sung Ju Hong ${ }^{3 凶}$ \& \\ Young-Mi Bahk ${ }^{1,2 \bowtie}$
}

We investigate conducting characteristics of biochar derived from the pyrolysis of a paper at terahertz frequencies. Paper is annealed under temperatures ranging from 600 to $1000^{\circ} \mathrm{C}$ to modify structural and electrical properties. We experimentally observe that the terahertz conductivity increases above $10^{2} \mathrm{~S} / \mathrm{m}$ as the annealing temperature increases up to $800^{\circ} \mathrm{C}$. From structural characterization using energy-dispersive X-ray spectroscopy, Fourier-transform infrared spectroscopy, Raman spectroscopy, $X$-ray diffraction, and X-ray photoelectron spectroscopy, we confirm that more graphitic biochars are produced in high annealing temperature, in agreement with the improvement of terahertz conductivity. Our results show that biochar can be a highly promising candidate to be used in paperbased devices operating at terahertz frequencies.

Paper is a cost-effective, abundant, disposable, and flexible material, therefore it is widely used in qualitative and simple diagnoses such as litmus tests ${ }^{1}$ and pregnancy tests ${ }^{2}$. Despite the advantages, poor electrical conductivity prevents it from being a sensing platform. Biochar obtained by annealing without oxygen can be a breakthrough for inducing electrical properties. Furthermore, its chemical stability under ambient conditions leads to various applications such as supercapacitor ${ }^{3}$, sensor ${ }^{4}$, water purification ${ }^{5}$, and conducting ink ${ }^{6}$. Therefore, the electrical evaluation of the biochar is of significance in the aspect of both fundamental and applicable points of view.

Terahertz time-domain spectroscopy is a representative tool for characterizing the electrical properties of material ranging from metal to dielectric in a non-contact manner. Especially, the terahertz wave can easily penetrate dielectric materials which are opaque in the visible and near-infrared regime. Therefore, various studies related to paper-based devices have also been conducted in terahertz optics; e.g., terahertz zone plate ${ }^{7}$, terahertz photonic $\mathrm{crystal}^{8}$, and terahertz metamaterial ${ }^{9,10}$. However, the previous works require an additional metal patterning process to realize terahertz devices on the paper-based substrates. Such a process generally needs expensive ingredients and generates pollutants, which contradicts using paper-based substrates as an inexpensive and eco-friendly sensing platform. In that regard, investigating the biochar in the terahertz regime is attractive to search low cost and eco-friendly materials.

Even though the biochars can be a potential candidate as an alternative, conductive material at the terahertz frequencies, there are few studies on their optical properties. Lepodise et al. investigated the absorption characteristics of aromatic carbon structures in biochars in the regime of $10 \mathrm{THz}^{11}$. In addition, Pogson et al. investigated the capability of the terahertz spectroscopy to detect or screen the various biochar components ${ }^{12}$. However, the conducting characteristics of biochar at the low terahertz frequency range (below $2 \mathrm{THz}$ ) are not studied yet. Since extensive studies for device application in the frequency regime are being actively conducted, investigating the terahertz electrical conductivity of biochar in that range is of significance.

Here, we investigate the conducting characteristics of the biochar at low terahertz frequencies $(0.7-1.5 \mathrm{THz})$. The biochar is prepared by annealing under a nitrogen $\left(\mathrm{N}_{2}\right)$ atmosphere at various temperatures. We confirm that the annealing process improves electrical conductivity in terms of terahertz time-domain spectroscopy. By harnessing structural analysis, we reveal that the origin of electrical conductivity enhancement of the biochar. We attribute the enhancement to the crystallization of carbon elements in the fibril network.

\footnotetext{
${ }^{1}$ Intelligent Sensor Convergence Research Center (ISCRC), Incheon National University, 22012 Incheon, Republic of Korea. ${ }^{2}$ Department of Physics, Incheon National University, 22012 Incheon, Republic of Korea. ${ }^{3}$ Division of Science Education, Kangwon National University, 24341 Chuncheon, Republic of Korea. ${ }^{4}$ These authors contributed equally: Woongkyu Park and Hyuntae Kim. ${ }^{\boxplus}$ email: sbchoi@inu.ac.kr; sjh@kangwon.ac.kr; ymb@ inu.ac.kr
} 

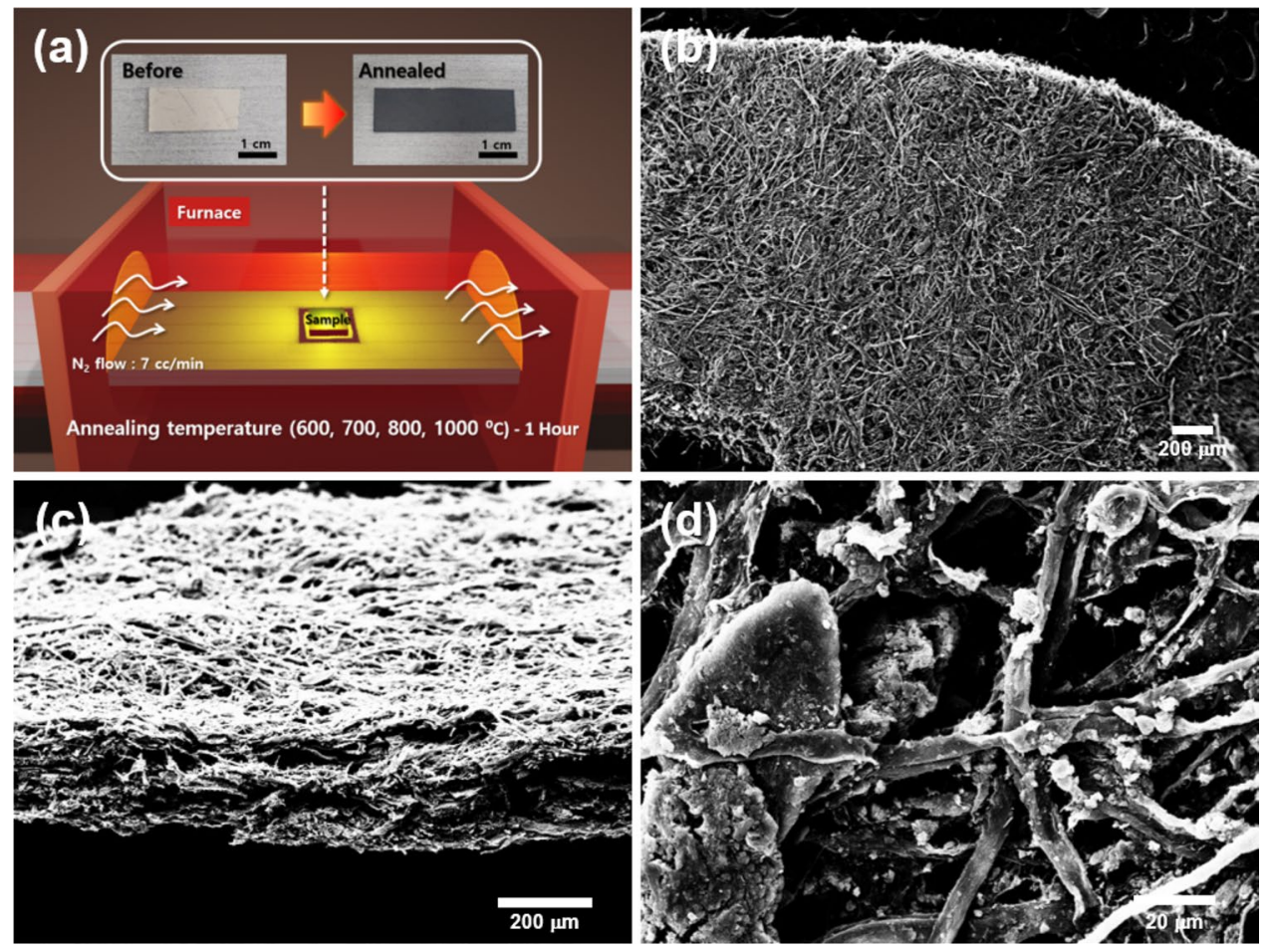

Figure 1. (a) Schematic image for annealing the paper substrate with various temperatures in $\mathrm{N}_{2}$ environment. Inset is photograph of the biochar before and after annealing [drawn by Blender (ver. 2.81) (https://www.blend er.org/download/releases/2-81)]. (b-d) SEM images of the annealed biochar with $600{ }^{\circ} \mathrm{C}$. (b,c) are top- and sideview of the biochar, respectively. (d) Fibril network and $\mathrm{CaCO}_{3}$ on the network are presented.

Results and discussion. The biochar sample was obtained by pyrolysis of a paper as shown in Fig. 1a. Detailed information on sample preparation is provided in "Methods" section. Figure $1 \mathrm{~b}-\mathrm{d}$ show scanning electron microscopy (SEM) images of annealed biochar sample at $600^{\circ} \mathrm{C}$, where the fibril network in the biochar is clearly shown. Note that the biochar can also be made as a powder on rigid substrates for practical applications such as terahertz shielding and metamaterials based on desired patterned conducting materials, as shown in Supplementary Information (Figs. S1, S2). We measured the terahertz transmission spectra and extracted frequency-dependent electrical conductivity of the biochar using a homemade terahertz time-domain spectroscopy system (Fig. 2a-e). The terahertz transmission and conductivity of the paper without annealing were also measured as a control experiment (black line). In Fig. 2b, one can check that the transmitted amplitude decreases as the annealing temperature increases. This phenomenon was also clearly shown in biochar powder samples, confirmed by terahertz imaging (Fig. S2). Especially, when the annealing temperature reaches 900 and $1000{ }^{\circ} \mathrm{C}$, the transmitted amplitude is indistinguishable from the noise. Therefore, data only up to $800{ }^{\circ} \mathrm{C}$ were used for subsequent analysis. Normalized amplitude and phase of Fourier transformed data were shown in Fig. 2c. Still, the normalized amplitude decreases as the annealing temperature increases. We note that resonances due to the absorption of low-energy photons, such as intermolecular vibrations, were not observed. Since the terahertz transmission is sensitive to the electrical conductivity of a material, it can be inferred that the decrease in the transmission is due to an increase in the electrical conductivity of the material. This hypothesis can be confirmed directly using the equations obtained in "Methods" section. Obtained terahertz electrical conductivity was shown in Fig. 2d,e. Surprisingly, the terahertz conductivity of the biochar increased exponentially with increasing annealing temperature. When the annealing temperature was $600{ }^{\circ} \mathrm{C}$, the electrical conductivity of the biochar was only a few $\mathrm{S} / \mathrm{m}$. However, as the annealing temperature increased up to $700{ }^{\circ} \mathrm{C}$ and $800{ }^{\circ} \mathrm{C}$, the electrical conductivity increased significantly to the order of $10 \mathrm{~S} / \mathrm{m}$ and $10^{2} \mathrm{~S} / \mathrm{m}$.

To understand the origin of becoming a conducting material, now we move on to elemental and structural analysis for supporting the electrical results. First of all, we carried out an energy-dispersive X-ray spectroscopy (EDS) experiment as shown in Fig. 3. It was confirmed that the constituent elements of the biochar are mainly carbon, oxygen, and calcium. Figure 3b-d show the EDS mapping image for each element, corresponding to the SEM image shown in Fig. 3a. The distribution of carbon mainly overlapped with the fibril network of the biochar as in Fig. 3a. On the other hand, the distribution of calcium was mainly overlapped with the distribution of particles shown in Fig. 3a (red-dotted circle). Oxygen was distributed relatively evenly. The observation is consistent with the fact that paper is composed of various materials such as cellulose ${ }^{13}$ and $\mathrm{CaCO}_{3}{ }^{14}$ (which is commonly used as a fertilizer), and these are mainly composed of carbon, oxygen, and calcium. That is, the difference in the distribution of each element can be understood as a result of the different distribution of substances (cellulose, $\mathrm{CaCO}_{3}$, etc.). 
(a)

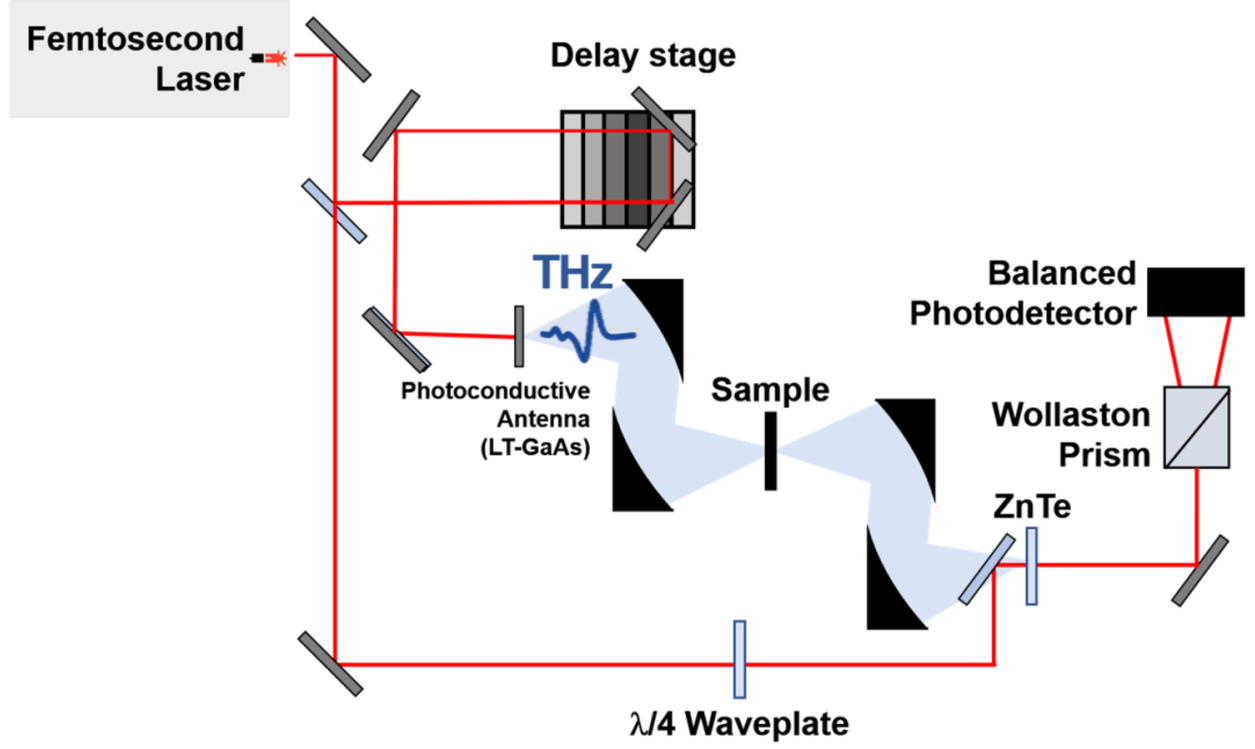

(b)

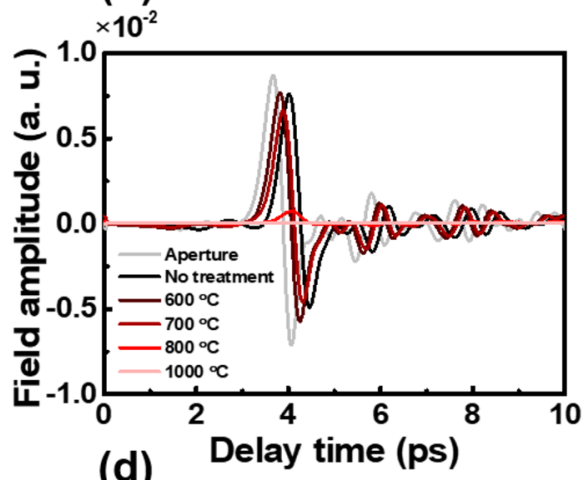

(d)

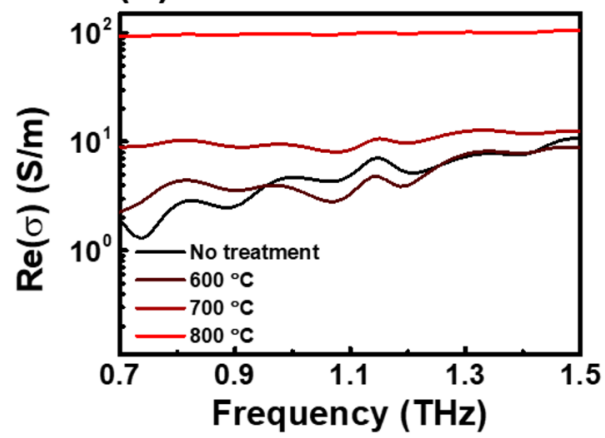

(c)
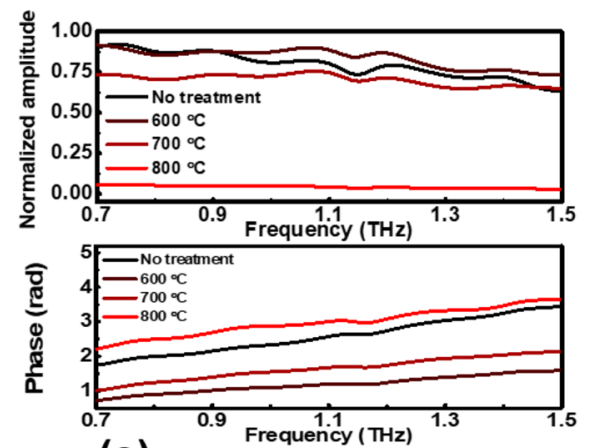

(e)

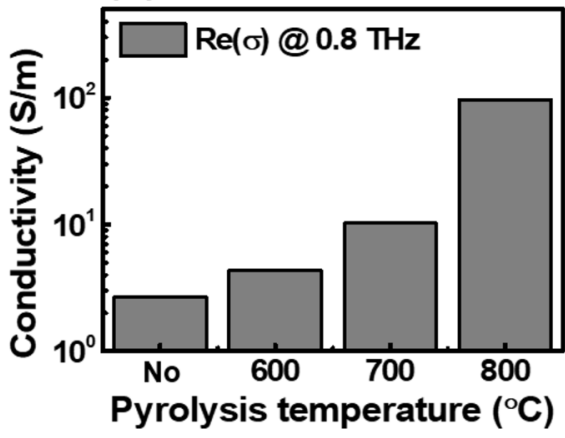

Figure 2. Terahertz time-domain spectroscopy of the paper substrate with and without annealing. (a) Experimental setup for terahertz time-domain spectroscopy. (b) Time trace of terahertz transmitted amplitude. (c) Normalized amplitude and phase of Fourier transformed data. (d) Frequency-dependent optical conductivity. (e) Summary of optical (at $0.8 \mathrm{THz}$ ) conductivity with annealing temperature.

The relative weight concentrations of each element in the biochar are summarized in Fig. 3e and Table 1. After the annealing process, the overall concentration of carbon and calcium increased, while the concentration of oxygen decreased. This can be understood through the process of carbonization. During the pyrolysis process, the moisture in the paper substrate volatilizes ${ }^{6}$. Also, cellulose begins to degrade at $315-400{ }^{\circ} \mathrm{C}^{15}$. At this temperature, the oxygen concentration becomes lower and the concentrations of carbon and calcium become relatively higher. It is noteworthy that the carbon (calcium) concentration gradually decreases (increases) as the annealing temperature increases. As the degradation of cellulose continues, all the volatile materials ( $\operatorname{such}$ as $\mathrm{CO}_{2}$, $\mathrm{CH}_{4}, \mathrm{CO}$, etc.) also begin to be removed ${ }^{6,15}$. Some carbon disappears in this situation, but calcium still remains. 

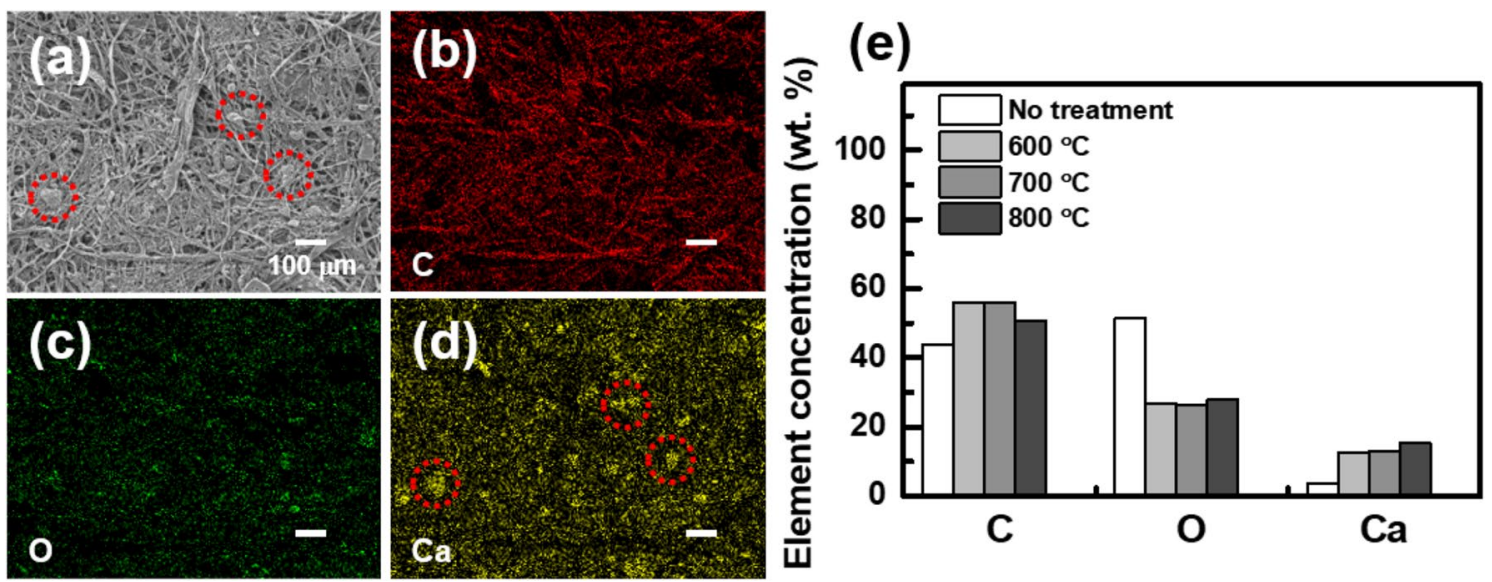

Figure 3. (a) SEM image and the corresponding EDS results for (b) carbon, (c) oxygen, and (d) calcium. The fibril structure matches with carbon distribution. The red-dotted circles in (a) and (d) show the overlap of the particles and calcium distribution. (e) Summary for the annealing-temperature-dependent element concentration.

\begin{tabular}{|c|c|c|c|c|c|c|c|c|}
\hline \multirow[b]{2}{*}{ Pyrolysis temperature } & \multicolumn{2}{|l|}{ C } & \multicolumn{2}{|l|}{ o } & \multicolumn{2}{|l|}{$\mathrm{Mg}$} & \multicolumn{2}{|l|}{ Al } \\
\hline & wt $\%$ & at $\%$ & wt $\%$ & at $\%$ & wt $\%$ & at $\%$ & wt\% & at $\%$ \\
\hline No treatment & 43.8 & 52.11 & 51.55 & 46.04 & & & 0.80 & 0.42 \\
\hline $600^{\circ} \mathrm{C}$ & 56.06 & 68.47 & 26.54 & 24.33 & 0.25 & 0.15 & 2.75 & 1.49 \\
\hline $700^{\circ} \mathrm{C}$ & 56.03 & 68.58 & 26.2 & 24.08 & 0.27 & 0.16 & 2.85 & 1.55 \\
\hline $800^{\circ} \mathrm{C}$ & 50.74 & 64.30 & 27.94 & 26.58 & 0.34 & 0.21 & 3.25 & 1.84 \\
\hline \multirow[t]{2}{*}{$1000^{\circ} \mathrm{C}$} & 56.02 & 69.18 & 24.49 & 22.71 & 0.37 & 0.23 & 2.8 & 1.54 \\
\hline & \multicolumn{2}{|l|}{$\mathrm{Si}$} & \multicolumn{2}{|l|}{ S } & \multicolumn{2}{|l|}{$\mathrm{Ca}$} & & \\
\hline Pyrolysis temperature & $\mathbf{w t} \%$ & at $\%$ & wt $\%$ & at $\%$ & wt $\%$ & at $\%$ & & \\
\hline No treatment & 0.39 & 0.20 & & & 3.46 & 1.23 & & \\
\hline $600^{\circ} \mathrm{C}$ & 1.61 & 0.84 & 0.4 & 0.18 & 12.40 & 4.54 & & \\
\hline $700^{\circ} \mathrm{C}$ & 1.63 & 0.85 & & & 13.02 & 4.77 & & \\
\hline $800^{\circ} \mathrm{C}$ & 1.83 & 0.99 & 0.44 & 0.21 & 15.44 & 5.86 & & \\
\hline $1000^{\circ} \mathrm{C}$ & 1.71 & 0.90 & 0.35 & 0.16 & 14.26 & 5.28 & & \\
\hline
\end{tabular}

Table 1. EDS data of biochar samples with different pyrolysis temperature.

This process becomes faster with higher annealing temperatures. Consequently, the concentration of carbon slightly decreases, and the calcium concentration increases in the annealing process with higher temperatures.

Based on the data in Table 1, we measured and analyzed the X-ray diffraction (XRD), Fourier-transform infrared spectroscopy (FT-IR), Raman, and X-ray photoelectron spectroscopy (XPS) spectra as shown in Fig. 4. Figure 4a shows the XRD data of each sample. In the case of the paper substrate, only peaks related to cellulose ${ }^{16}$ and $\mathrm{CaCO}_{3}{ }^{17}$ were observed. On the other hand, in the case of the $600{ }^{\circ} \mathrm{C}$-annealed-sample, no cellulose-related picks were observed, while $\mathrm{CaCO}_{3}$-related picks were still observed. When the annealing temperature increased up to $700{ }^{\circ} \mathrm{C}$ or $800^{\circ} \mathrm{C}$, the calcium-carbonate-related peaks disappeared, and instead, calcium-hydroxide-related peaks were observed ${ }^{18}$. Also, no carbon-related peaks were observed. The thermal decomposition of $\mathrm{CaCO}_{3}$ is known to be initiated around $700-800{ }^{\circ} \mathrm{C}^{19}$. At this temperature, $\mathrm{CaCO}_{3}$ releases carbon dioxide and becomes calcium oxide ${ }^{20}$. After that, calcium oxide is combined with water vapor in the air to become calcium hydroxide ${ }^{19}$. During this process, some carbon is volatilized, reducing the relative weight concentration in the entire sample. These XRD results are consistent with the EDS results summarized in Fig. 3e. We may attribute the intensity of the carbon-related peak, which is relatively weak compared to that of the calcium-related materials, to the low crystallinity of the pyrolyzed paper.

Figure $4 \mathrm{~b}$ shows the FT-IR spectra of each sample. It can be seen that the spectra of the biochar samples and the paper substrate are significantly different. Two broad absorption bands are observed in the spectrum of the paper substrate: $3730-2800 \mathrm{~cm}^{-1}$ and $1700-800 \mathrm{~cm}^{-1}$. On the other hand, all these peaks disappear from the spectra of the biochar samples. In the biochar samples, peaks are mainly observed at $1400 \mathrm{~cm}^{-1}$ and $875 \mathrm{~cm}^{-1}$, and if the annealing temperature exceeds $600{ }^{\circ} \mathrm{C}$, a small peak is also observed near $3642 \mathrm{~cm}^{-1}$. In the spectrum of the paper substrate, the absorption peak at $3340 \mathrm{~cm}^{-1}$ is mainly due to the $\mathrm{O}-\mathrm{H}$ stretch bond, while the peak at $2898 \mathrm{~cm}^{-1}$ is attributed to the $\mathrm{C}-\mathrm{H}$ stretch bond in cellulose $\mathrm{e}^{21}$. In addition, the peak at $1162 \mathrm{~cm}^{-1}, 1106 \mathrm{~cm}^{-1}$, $1049 \mathrm{~cm}^{-1}$, and $1029 \mathrm{~cm}^{-1}$ are attributed to the asymmetric $\mathrm{C}-\mathrm{O}-\mathrm{C}$ bridge stretch bond ${ }^{22}$, anhydroglucose ring $\mathrm{g}^{22}$, $\mathrm{C}-\mathrm{O}-\mathrm{C}$ pyranose ring skeletal vibration ${ }^{23}$, and $\mathrm{C}-\mathrm{O}$ stretch bond ${ }^{24}$, respectively. These peaks indicate that the 
(a)
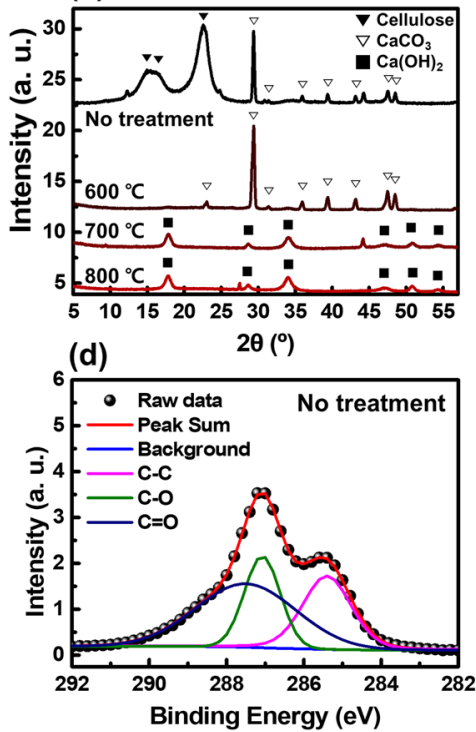

(b)

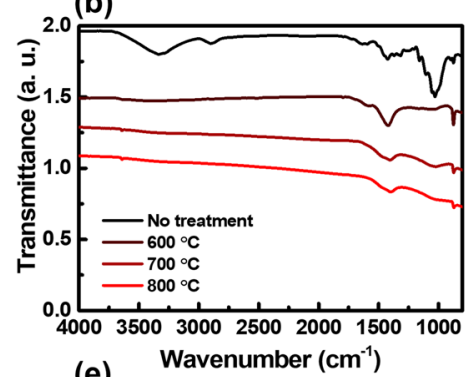

(e)

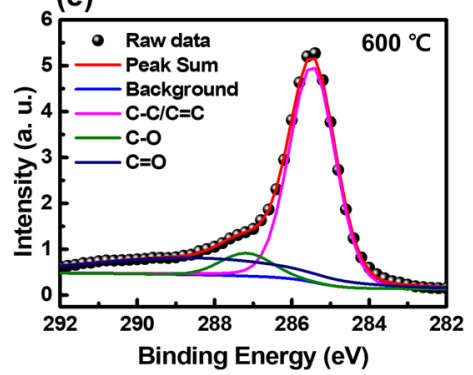

(c)

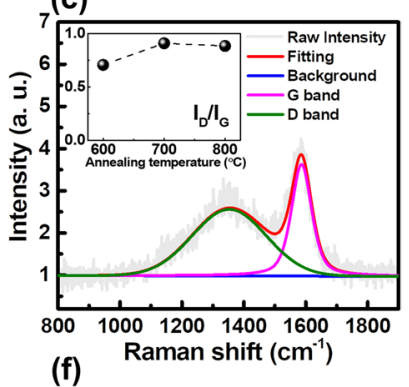

(f)

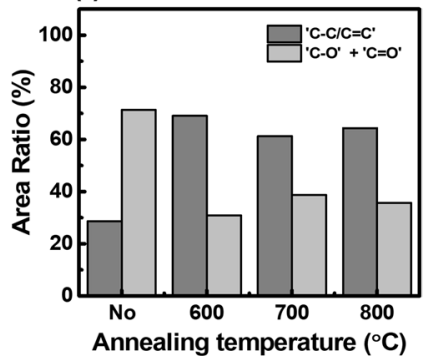

Figure 4. Structural analysis such as (a) XRD, (b) FT-IR, (c) Raman spectroscopy, and (d-f) XPS results, respectively.

paper substrate is mainly composed of cellulose. In the spectrum of the biochar samples, the broad absorption peak around $1400 \mathrm{~cm}^{-1}$ and a sharp peak at $874 \mathrm{~cm}^{-1}$ are attributed to the vibrations of $\mathrm{CO}_{3}^{-225}$. The small and sharp pick at $3642 \mathrm{~cm}^{-1}$ is due to the $\mathrm{O}-\mathrm{H}$ stretching vibration in calcium hydroxide ${ }^{26}$. Legodi et al. demonstrated that the peak at $874 \mathrm{~cm}^{-1}$ in the FT-IR spectra could be a quantitative tool for measuring the ratio of $\mathrm{CaCO}_{3} /$ $\mathrm{Ca}(\mathrm{OH})_{2}$ mixtures $^{26}$. According to previous literature ${ }^{26}$, the peak intensity increases as the proportion of $\mathrm{CaCO}_{3}$ in the mixture increases. In our experiments, the peak intensity gradually decreases as the annealing temperature increases. That is, as the annealing temperature increases, the rate at which $\mathrm{CaCO}_{3}$ is decomposed into calcium hydroxide increases. This argument can also be supported by an increase of the peak intensity at $3642 \mathrm{~cm}^{-1}$, which is attributed to the $\mathrm{O}-\mathrm{H}$ stretching vibration of the calcium hydroxide. This result is consistent with the XRD spectra shown in Fig. 4a. It should be noted that the baseline of the FT-IR spectra gradually slopes down as the annealing temperature increases. This is a unique property observed when carbon materials such as biochar and carbon black are measured by attenuated total reflection-Fourier transform infrared spectroscopy (ATR-FTIR) ${ }^{27}$. When using ATR-FTIR, light absorption from carbon materials becomes greater with deeper light penetration at the lower wavenumber. This trend becomes greater as the absorption coefficient (or real part of the complex conductivity $\operatorname{Re}(\tilde{\sigma}(\omega)))$ increases. That is, it shows that the optical conductivity of the biochar increases as the annealing temperature increases.

Figure $4 \mathrm{c}$ exhibits the Raman spectrum of $600^{\circ} \mathrm{C}$-annealed sample. In the case of the paper substrate, no significant spectrum was obtained. While, typical Raman spectra of carbon-based materials were observed in the biochar samples: e.g. $D$ and $G$ band near $1353 \mathrm{~cm}^{-1}$ and $1587 \mathrm{~cm}^{-1}$, respectively. The intensity ratio between $\mathrm{D}$ and $\mathrm{G}$ band in the inset of Fig. $4 \mathrm{c}$ can be employed to estimate the crystallinity degree of the carbon-based materials. The observed $\mathrm{I}_{\mathrm{D}} / \mathrm{I}_{\mathrm{G}}<1$ for all annealed cases indicates that the annealing process promotes crystallization of the paper substrate ${ }^{28}$.

Finally, we performed XPS experiments as shown in Fig. 4d-f. Figure 4d,e show the XPS data of the paper substrate and $600^{\circ} \mathrm{C}$-annealed sample, respectively. As mentioned above, paper substrates are mainly composed of cellulose. Therefore, it can be seen that the XPS spectrum of the paper substrate is consistent with the XPS spectrum of the cellulose $\mathrm{e}^{29-32}$. When the paper substrate is pyrolyzed to become biochar, the ratio of $\mathrm{C}-\mathrm{O}$ bonds and $\mathrm{C}=\mathrm{O}$ bonds decreases, and the ratio of $\mathrm{C}-\mathrm{C}$ bonds or $\mathrm{C}=\mathrm{C}$ bonds increases by the carbonization process. The ratio of each bond depending on the annealing temperature is summarized in Fig. $4 \mathrm{f}$. It is clearly shown that the ratio of $\mathrm{C}-\mathrm{C}$ or $\mathrm{C}=\mathrm{C}$ bond increased significantly in all biochar samples. That is, it can be seen that the carbon element remaining after the cellulose is pyrolyzed undergoes a crystallization process. This result is consistent with the Raman spectra shown in Fig. 4c. To summarize, by examining the various spectroscopic properties of the biochar, we have shown that the terahertz conductivity enhancement of the biochar was mainly originated from the pyrolysis of cellulose and the crystallization of carbon elements.

\section{Conclusion}

In conclusion, we have demonstrated that the terahertz electrical conductivity of biochar obtained from paper substrate increases dramatically with increasing the annealing temperature. It was confirmed that the origin of the conductivity enhancement is mainly due to the crystallization of carbon elements after the cellulose in the paper substrate is thermally decomposed. As the annealing temperature increases, pyrolysis and crystallization proceed faster, resulting in higher electrical conductivity of the biochar. Our research shows that biochar, an eco-friendly, and sustainable material has a great potential to be used in paper-based devices operating at terahertz frequencies. 


\section{Methods}

Materials. We used Elephant Poopoopaper (PoopoopaperTM) which is made in a chemical-free way and is environmentally friendly. The paper was placed in a furnace under a $\mathrm{N}_{2}$ environment and the furnace was heated up to $600,700,800,900$, and $1000^{\circ} \mathrm{C}$ at a rate of $5^{\circ} \mathrm{C} / \mathrm{min}$, respectively. Each sample was annealed for 1 hour. After the annealing process, the color changed from white to black as shown in the inset of Fig. 1a. No particular difference in surface flatness was found, as shown in Supplementary Information (Fig. S3). Note that calcium carbonate $\left(\mathrm{CaCO}_{3}\right)$ particles are also shown on the network, which was discussed in "Results and discussion" section.

Terahertz time-domain spectroscopy. We used Ti:sapphire femtosecond laser and low-temperaturegrown GaAs photoconductive antenna to generate terahertz pulses. The center wavelength, the repetition rate, and the pulse width of the laser are $800 \mathrm{~nm}, 80 \mathrm{MHz}$, and $100 \mathrm{fs}$, respectively. The terahertz pulses are focused with parabolic mirrors and normally incident on the biochar. Transmitted terahertz waves are collected by parabolic mirrors and detected via electro-optic sampling method using a (110)-oriented 1-mm-thick ZnTe crystal. The time-domain waveforms are converted to frequency spectra using a numerical Fourier transform. The terahertz spectra of the biochar are normalized to the spectra from the air.

Calculations of the complex refractive index and the complex conductivity. The relation between the normalized spectra and the complex refractive index of the biochar can be expressed as follows ${ }^{33}$ :

$$
\tilde{t}(\omega)=\rho(\omega) \cdot \exp [i \theta(\omega)]=\frac{4 \tilde{n}(\omega) \cdot \exp \left[i(\tilde{n}(\omega)-1) k_{0} h\right]}{(\tilde{n}(\omega)+1)^{2}}
$$

where $\rho(\omega)$ is an amplitude, $\theta(\omega)$ is a phase of the normalized spectra, $\tilde{n}(\omega)=n(\omega)+i \kappa(\omega)$ is a complex refractive index of the biochar, $\omega$ is the angular frequency, $c$ is light velocity, and $h$ is a thickness of the biochar, respectively. From this equation, we extracted the complex refractive index $\tilde{n}(\omega)$ by using the numerical method. Then, from the relation ${ }^{34}$

$$
\tilde{n}(\omega)=\sqrt{\tilde{\varepsilon}(\omega)}=\sqrt{1+\frac{i \tilde{\sigma}(\omega)}{\varepsilon_{0} \omega}}
$$

where $\tilde{\varepsilon}(\omega)$ is a complex dielectric function, $\varepsilon_{0}$ is a vacuum permittivity, $\tilde{\sigma}(\omega)$ is a complex conductivity, $\tilde{\sigma}(\omega)$ can be expressed as $\sigma(\omega)=-i \varepsilon_{0} \omega\left(\tilde{n}^{2}(\omega)-1\right)$. Therefore, the real part of the terahertz conductivity $\operatorname{Re}(\tilde{\sigma}(\omega))$ is determined by $\operatorname{Re}(\tilde{\sigma}(\omega))=2 n(\omega) \kappa(\omega) \varepsilon_{0} \omega$.

Structural characterization. The morphology of the biochar samples and their energy-dispersive X-ray spectra (EDS) were obtained using field emission scanning electron microscopy (JSM-7800F, JEOL). The crystallinity and compositions of the biochar were characterized by X-ray diffraction (XRD) using a high-resolution $\mathrm{X}$-ray diffractometer (SmartLab, Rigaku) with $\mathrm{Cu} \mathrm{Ka}$ radiation. Elemental compositions of the biochar were also analyzed using X-ray photoelectron spectroscopy (XPS) (PHI 5000 VersaProbe II, ULVAC-PHI). In addition, Fourier-transform infrared spectroscopy (FT-IR) (VERTEX 80v and Hyperion 2000, Bruker) and Raman spectroscopy (Xper Ram 200, Nanobase) using a $532 \mathrm{~nm}$ laser were also performed to analyze the molecular structure of the biochar.

Received: 20 May 2021; Accepted: 1 September 2021

Published online: 16 September 2021

\section{References}

1. Crosland, M. P. Gay-Lussac (Cambridge University Press, 1978).

2. Macgillivray, I. \& Tovey, J. E. A study of the serum protein changes in pregnancy and toxaemia, using paper strip electrophoresis. J. Obstet. Gynaecol. Br. Emp. 64, 361-364 (1957).

3. Abioye, A. M. \& Ani, F. N. Recent development in the production of activated carbon electrodes from agricultural waste biomass for supercapacitors: A review. Renew. Sustain. Energy Rev. 52, 1282-1293 (2015).

4. Nery, E. W. \& Kubota, L. T. Sensing approaches on paper-based devices: A review. Anal. Bioanal. Chem. 405, 7573-7595 (2013).

5. Dehkhoda, A. M., Gyenge, E. \& Ellis, N. A novel method to tailor the porous structure of KOH-activated biochar and its application in capacitive deionization and energy storage. Biomass Bioenergy 87, 107-121 (2016).

6. Fingolo, A. C. et al. Boosting electrical conductivity of sugarcane cellulose and lignin biocarbons through annealing under isopropanol vapor. ACS Sustain. Chem. Eng. 8, 7002-7010 (2020).

7. Ivaškevičiūtè-Povilauskienè, R. et al. Flexible materials for terahertz optics: advantages of graphite-based structures. Opt. Mater. Express 9, 4438 (2019).

8. Zhang, W., Lin, X., Jin, Z., Ma, G. \& Zhong, M. Design, fabrication and transmitted properties of terahertz paper photonic crystals. Opt. Express 21, 27622 (2013).

9. Tao, H. et al. Metamaterials on paper as a sensing platform. Adv. Mater. 23, 3197-3201 (2011).

10. Sadeqi, A., Nejad, H. R. \& Sonkusale, S. Low-cost metamaterial-on-paper chemical sensor. Opt. Express 25, 16092 (2017).

11. Lepodise, L. M. et al. Terahertz spectroscopy of biochars and related aromatic compounds. J. Infrared Milli. Terahz. Waves 37, 1158-1165 (2016).

12. Pogson, E. M., et al. Detection of biochar components for soil fertility using THz-TDS. In 35th International Conference on Infrared, Millimeter, and Terahertz Waves (IRMMW-THz), Rome, Italy (2010). 
13. Sjöström, E. \& Westermark, U. Chemical Composition of Wood and Pulps: Basic Constituents and Their Distribution. In Analytical Methods in Wood Chemistry, Pulping, and Papermaking (eds Sjöström, E. \& Alén, R.) 1-19 (Springer, 1999).

14. He, M., Cho, B. U. \& Won, J. M. Effect of precipitated calcium carbonate-cellulose nanofibrils composite filler on paper properties. Carbohydr. Polym. 136, 820-825 (2016).

15. Deng, J., Li, M. \& Wang, Y. Biomass-derived carbon: Synthesis and applications in energy storage and conversion. Green Chem. 18, 4824-4854 (2016).

16. Gong, J., Li, J., Xu, J., Xiang, Z. \& Mo, L. Research on cellulose nanocrystals produced from cellulose sources with various polymorphs. RSC Adv. 7, 33486-33493 (2017).

17. Kontoyannis, C. G. \& Vagenas, N. V. Calcium carbonate phase analysis using XRD and FT-Raman spectroscopy. Analyst 125, 251-255 (2000).

18. Kalinkin, A. M., Kalinkina, E. V., Zalkind, O. A. \& Makarova, T. I. Chemical interaction of calcium oxide and calcium hydroxide with CO2 during mechanical activation. Inorg. Mater. 41, 1073-1079 (2005).

19. Karunadasa, K. S. P., Manoratne, C. H., Pitawala, H. M. T. G. A. \& Rajapakse, R. M. G. Thermal decomposition of calcium carbonate (calcite polymorph) as examined by in-situ high-temperature X-ray powder diffraction. J. Phys. Chem. Solids 134, 21-28 (2019).

20. Sanders, J. P. \& Gallagher, P. K. Kinetic analyses using simultaneous TG/DSC measurements. Thermochim. Acta 388, 115-128 (2002).

21. Hospodarova, V., Singovszka, E. \& Stevulova, N. Characterization of cellulosic fibers by FTIR spectroscopy for their further implementation to building materials. Am. J. Anal. Chem. 09, 303-310 (2018).

22. Robles, E., Urruzola, I., Labidi, J. \& Serrano, L. Surface-modified nano-cellulose as reinforcement in poly(lactic acid) to conform new composites. Ind. Crops Prod. 71, 44-53 (2015).

23. Chen, W. et al. Thermo-responsive cellulose-based material with switchable wettability for controllable oil/water separation. Polymers 10, 20 (2018).

24. Xu, F., Yu, J., Tesso, T., Dowell, F. \& Wang, D. Qualitative and quantitative analysis of lignocellulosic biomass using infrared techniques: A mini-review. Appl. Energy 104, 801-809 (2013).

25. Jovanovski, G., Stefov, V., Šoptrajanov, B. \& Boev, B. Minerals from Macedonia. IV. Discrimination between some carbonate minerals by FTIR spectroscopy. Neues Jahrb. Mineral. Abhandlungen 177, 241-253 (2002).

26. Legodi, M. A., De Waal, D., Potgieter, J. H. \& Potgieter, S. S. Rapid determination of CaCo3 in mixtures utilising FT-IR spectroscopy. Miner. Eng. 14, 1107-1111 (2001).

27. Manohar, N. et al. A unique application of the second order derivative of FTIR-ATR spectra for compositional analyses of natural rubber and polychloroprene rubber and their blends. Polym. Test. 62, 447-453 (2017).

28. Ferrari, A. \& Robertson, J. Interpretation of Raman spectra of disordered and amorphous carbon. Phys. Rev. B 61, 14095-14107 (2000).

29. Bastidas, J. C. et al. Chemical force microscopy of cellulosic fibers. Carbohydr. Polym. 62, 369-378 (2005).

30. Kuzmenko, V. et al. Cellulose-derived carbon nanofibers/graphene composite electrodes for powerful compact supercapacitors. RSC Adv. 7, 45968-45977 (2017).

31. Serbanescu, O. S., Pandele, A. M., Miculescu, F. \& Voicu, S. I. Synthesis and characterization of cellulose acetate membranes with self-indicating properties by changing the membrane surface color for separation of Gd(III). Coatings 10, 20 (2020).

32. Wang, Q., Xie, D., Chen, J., Liu, G. \& Yu, M. Superhydrophobic paper fabricated via nanostructured titanium dioxide-functionalized wood cellulose fibers. J. Mater. Sci. 55, 7084-7094 (2020).

33. Born, M. \& Wolf, E. Chapter XIII. Optics of Metals. In Principles of Optics: Electromagnetic Theory of Propagation, Interference and Diffraction of Light (eds Born, M. et al.) 611-664 (Cambridge University Press, 1999).

34. Maier, S. A. Electromagnetics of metals. In Plasmonics: Fundamentals and Applications (ed. Maier, S. A.) 5-19 (Springer, 2007).

\section{Acknowledgements}

This research was supported by Basic Science Research Program through the National Research Foundation of Korea (NRF) funded by the Ministry of Education (2020R1I1A1A01070240) and Research Assistance Program (2019) in the Incheon National University, and 2020 Research Grant from the Kangwon National University. The authors acknowledge Won Taek Jung, Hyun-Seok Jang, and Byung Hoon Kim for annealing process.

\section{Author contributions}

The experimental concept was conceived by W.P., H.K., and Y.-M.B. H.P. performed THz-TDS. W.P. and H.K. performed EDS, XRD, FT-IR, Raman spectroscopy, and XPS. W.P., H.K., S.C., S.J.H., and Y.-M.B. analyzed the experimental data. The manuscript was written by W.P., H.K., S.J.H., and Y.-M.B. based on discussions with S.C. All authors discussed the results and commented on the manuscript.

\section{Competing interests}

The authors declare no competing interests.

\section{Additional information}

Supplementary Information The online version contains supplementary material available at https://doi.org/ 10.1038/s41598-021-98009-5.

Correspondence and requests for materials should be addressed to S.C., S.J.H. or Y.-M.B.

Reprints and permissions information is available at www.nature.com/reprints.

Publisher's note Springer Nature remains neutral with regard to jurisdictional claims in published maps and institutional affiliations. 
(c) (i) Open Access This article is licensed under a Creative Commons Attribution 4.0 International cc) License, which permits use, sharing, adaptation, distribution and reproduction in any medium or format, as long as you give appropriate credit to the original author(s) and the source, provide a link to the Creative Commons licence, and indicate if changes were made. The images or other third party material in this article are included in the article's Creative Commons licence, unless indicated otherwise in a credit line to the material. If material is not included in the article's Creative Commons licence and your intended use is not permitted by statutory regulation or exceeds the permitted use, you will need to obtain permission directly from the copyright holder. To view a copy of this licence, visit http://creativecommons.org/licenses/by/4.0/.

(C) The Author(s) 2021 